\title{
Improved Detection Limits for Fast Atom Bombardment Mass Spectrometry: A Study of Time-Dependent Desorption Using a Model Pulsed Bombardment Ionization Method
}

\author{
Andrew N. Tyler and Laura K. Romo \\ Department of Chemistry, Harvard University, Cambridge, Massachusetts, USA
}

\author{
Michael H. Frey, Brian D. Musselman, Jun Tamura, and Robert B. Cody \\ JEOL USA, Inc., Peabody, Massachusetts, USA
}

\begin{abstract}
Certain sample preparations for fast atom bombardment (FAB) yield an intense but brief pulse of sample ions at the onset of ionization. A model system is used to study this phenomenon. This system utilizes a conventional source of a constant flux of fast atoms and a probe that permits mechanical movement of the sample stage. This is used to simulate the effect of pulsing the atom beam. Experiments with model samples and selected ion monitoring detection show that, following bombardment initiation, sample ions are preferentially desorbed with respect to ions from the FAB matrix. Exhibition of preferential sample desorption depends upon the analyte behaving as a surfactant in the selected matrix. When used in conjunction with an array detector that permits data collection in a time-resolved manner, this system allows collection of useful mass spectra with significantly enhanced sensitivity compared to normal bombardment. When applied to the undecapeptide eledoisin (sequence pyro-EPSKDAFIGLM-NH ${ }_{2}, \mathrm{MW} 1187.6 \mathrm{Da}$ ) this novel methodology allows an improvement in detection limit of at least three to four orders of magnitude over that observed when using conventional continuous FAB and a point detector. (J Am Soc Mass Spectrom 1992, 3, 637-643)
\end{abstract}

$\mathrm{O}^{\mathrm{i}}$ ince its introduction [1], fast atom bombardment (FAB) has developed into a standard ionization method for the mass spectrometric analysis of involatile materials. A typical $\mathrm{FAB}$ analysis requires dispersion of the material to be analyzed in a low volatility solvent, such as glycerol, which is commonly known as a matrix. This preparation is normally subjected to a steady flux of rare gas atoms with a typical kinetic energy of 3 to $10 \mathrm{keV}$ and the ions so produced are mass analyzed. High-energy cesium ions may be used instead of atoms to effect desorption. This technique is commonly known as liquid secondary ion mass spectrometry (LSIMS) and yields similar mass spectra [2].

FAB mass spectra are usually characterized by background ions that produce a weak signal at each mass over the whole mass range. This background is usually referred to as "chemical noise" to distinguish it from the electronic noise common to all electronic

Address reprint requests to Andrew N. Tyler, Department of Chemistry, Harvard University, 12 Oxford Street, Cambridge, MA 02138. measurement techniques. In the presence of this chemical noise spectrum, to optimize sensitivity for FAB ionization one searches for the conditions under which the observed intensity ratio of analyte ion signals to chemical noise is maximized. This requires some insights into the mechanisms that are likely to control and regulate sample ion production.

One consequence of the sample preparation procedure is that the solution chemistry of the analyte in the chosen matrix is very important, because this will largely determine the quality of data that will be obtained. The factors involved in determining the FAB sensitivity for a given analyte in a particular matrix are complex. Some important factors appear to be the number of charged functional groups on the analyte, the material's hydrophobicity, its solubility in the chosen matrix, and the concentration of analyte in the matrix. In particular, the tendency of the analyte to behave as a surfactant in the matrix is one major factor in determining whether a material will give sensitive FAB spectra $[3,4]$. The role of surface activity has been extensively studied, particularly by Ligon and coworker [5-7]. 
Pulsed atom bombardment has been evaluated previously for enhanced sensitivity of tandem mass spectrometry on magnetic sector instruments [8]. These workers focused upon pulsed FAB as a method of enhancing protonated molecule intensities for subsequent collision-induced dissociation and photodissociation experiments on a tandem mass spectrometer. The experiments were conducted by pulsing the flow of bombarding gas into the discharge chamber of the atom gun. This methodology limited the maximum pulse repetition rate to one pulse every $5 \mathrm{~s}$. The total ion yields and ion currents following collision-induced dissociation increased by up to a factor of 15 compared to the yields for conventional FAB. This gain was attributed to a combination of reduced ion source pressure and increased primary neutral beam densities resulting from the method of pulsing. The enhanced desorption of analyte ions over matrix ions was not observed under the conditions used for these experiments.

In contrast, the experiments conducted in our laboratories have been directed toward the use of pulsed particle bombardment as a method for specifically enhancing the ratio of analyte ion abundances to matrix background ions for conventional mass spectrometry.

Pulsed beams have also been used for studies on other designs of mass spectrometer. Experiments with pulsed cesium ion beams and a tandem quadrupole ion cyclotron resonance mass spectrometer [9] indicate that the ion yields for picomole quantities of small proteins are greater than those obtained by conventional FAB. In these experiments the repetition rate of the pulses was $200 \mathrm{~Hz}$ and the enhanced ion yields are ascribed to the reduction in the ion source pressure resulting from the use of cesium ions instead of xenon gas.

Pulsed ion beams have been used to perform LSIMS experiments on time-of-flight mass spectrometers. Initial experiments utilized pulse frequencies of 50 and $100 \mathrm{~Hz}$ [10], while later experiments studied molecular signal intensities as a function of primary ion pulse frequency [11]. These experiments studied bombardment with primary ion beam pulses in a frequency range from 10 to $50 \mathrm{kHz}$ with pulse widths of $4 \mu \mathrm{s}$. These experiments showed a decrease in $[\mathrm{M}+\mathrm{H}]^{+}$ion intensities for the materials studied as the bombardment pulse frequency increased, an observation rationalized in terms of time required to repair the surface damage that resulted from the preceding bombardment pulse. While these workers suggested that rapid pulse repetition results in protonated molecule intensities more in agreement with bulk concentration than with surface concentration, no direct comparisons of analyte and matrix ion relative intensities were reported.

Musselman et al. [12], working with a conventional double-focusing instrument operating in the selected ion monitoring (SIM) mode, made the observation that certain sample preparations yield a large pulse of analyte ions at the onset of bombardment. In addition, the accompanying matrix ion signals were suppressed during this time period. The observed analyte ion pulse width was on the order of $200 \mathrm{~ms}$. In our initial experiments we were able to observe similar pulses and in some cases we were able to observe pulses of sample ions from samples that were too dilute to yield significant sample signals under normal bombardment conditions. These observations imply a possible method to improve FAB sensitivity for samples that yield these pulses. However, because the pulse duration is so short, the experiment is of little practical value when using mass spectrometers with point detectors. Scanning these instruments at a speed sufficient to collect a spectrum during the ion pulse is impractical. It is necessary to know the precise mass of the analyte ion prior to bombardment so that the instrument may be set to monitor that mass. This technique is useful for gaining insights into the physical mechanisms responsible for the pulsing behavior. but to make use of the pulsing effect for analytical work one requires a detector that will permit simultaneous detection of a wide mass range of ions in a time-resolved fashion.

The use of ion detection systems that, when placed along the focal plane of a magnetic instrument, will detect several ions with consecutive different masses simultaneously have been reported [13-15]. The development of these detectors has been primarily motivated by the fact that concurrent mass analysis allows a sensitivity enhancement for tandem mass spectrometry techniques, in which matrix background signals are often eliminated by the nature of the scan. These detectors have not yet found wide application with conventional double-focusing (i.e., two-sector) mass spectrometers because the major factor limiting sensitivity for normal mass spectra is chemical noise rather than electronic noise. Compared to conventional point detectors, array detectors can offer improved performance when ion statistics becomes the factor that limits sensitivity.

Array detectors record all the ions that fall within their operational mass range over some time period. Signals can be integrated for several seconds if the detector is not saturated by the arriving signals. The integrated data are subsequently transferred and logged by a data system. The detector is then refreshed to allow detection of the signals arriving in the next time window.

The transit time of $10-\mathrm{keV}$-energy ions passing through a double-focusing instrument is on a $\mathrm{mi}$ crosecond time scale. This period is short compared with that of the ion pulses evolved in the initial stages of bombardment, which occur on a millisecond time scale. Therefore the array detector potentially offers a method of recording spectra from the pulses of preferentially desorbed analyte ions.

\section{Experimental}

The mass spectrometer used for SIM experiments was a JEOL JMS-AX $505 \mathrm{H}$ double-focusing mass spectrom- 
eter. Instrument characteristics were: $6-\mathrm{keV}$ xenon atom beam, 5-mA atom gun emission current, $3-\mathrm{keV}$ ion source energy. The data were collected by a standard JEOL DA-5000 data system. To alternate between sample and matrix ions as rapidly as possible, peak tops, rather than peak profiles, were monitored in the SIM experiments.

The equipment used for these experiments was not designed to produce pulsed fast atom beams. To simulate a pulsed bombardment effect a $F A B$ insertion probe was used that allows the probe shaft, and hence the sample tip, to be manually rotated within the $F A B$ source. A double-sided tip was fitted to the probe so that either side of the tip could be exposed to the fast atom beam. Samples were prepared on one side of the probe tip. By rotating the probe the sample could be exposed to bombardment by a stable atom beam for a chosen interval, then rotated out of the bombarding beam for some period to allow the sample to equilibriate, then rotated into the beam again. Similar results were observed by either switching the $\mathrm{FAB}$ gun filament on and off or by regulating the xenon gas supply. However, these methods were found to be less reliable than rotating the probe, because the period required for the atom beam to stabilize is significant on the time scale of these experiments. Signals recorded in the SIM experiments were the molecular species for each analyte and the matrix-derived ion nearest in mass to the molecular signal. The data system was set to monitor each mass for $30 \mathrm{~ms}$ with a $10-\mathrm{ms}$ settling time between measurements. These cycle times were the fastest possible with the equipment used.

The mass spectrometer used for the array detector experiments was a JEOL JMS-HX 110 double-focusing mass spectrometer equipped with a prototype 3 -in. array detector. The array detector was placed at the focal plane of the mass spectrometer and consisted of a 3-in. microchannel plate (MCP), a phosphor-coated fiber-optic interface, and a 2-in. photodiode array. The fiber-optic element reduces the 3-in. image at the output of the MCP to a 2-in. image that is compatible with the dimensions of the photodiode array. This array detector follows the design of Hill et al. [15], and it can simultaneously detect approximately $10 \%$ of the mass range about a given center mass. Data acquisition was controlled by a Princeton Instruments ST120 Optical Multichannel Analyzer Controller interfaced to a Compaq 386/20 computer. The mass spectrometer control computer was used to adjust the magnetic sector field strength to the desired center mass. Data reduction and analyses were performed using SpectraCalc software (SpectraCalc, Salem, NH) written for the Compaq computer. The instrumental operating conditions were the same as for the SIM experiments except the ion source was operated at $10-\mathrm{keV}$ ion energy.

For the array detector experiments the data system was set to integrate signals from the array detector for $65 \mathrm{~ms}$ followed by a reset and refresh time of $35 \mathrm{~ms}$, for a total cycle time of $100 \mathrm{~ms}$. The sample was rotated to allow the xenon beam to impinge upon the sample for a brief interval, then rotated out of the atom beam for differing periods, lasting up to $20 \mathrm{~s}$, to allow the sample equilibrium solution condition to reestablish before the sample was once again rotated into the atom beam.

For all experiments samples were prepared by successive dilutions of a standard sample. Glycerol (Fisher Scientific, Fair Lawn, NJ), 3-nitrobenzyl alcohol (NBA) 98\% (Aldrich Chemical Co., Milwaukee, WI), thioglycerol, erythromycin, and eledoisin (Sigma Chemical Co., St. Louis, MO) were used as received. Samples were deposited as $1-\mu \mathrm{L}$ methanol or water solutions onto one side of a double-sided FAB tip that had been precoated with about $1 \mu \mathrm{L}$ of matrix. Samples were introduced and bombarded as described in the text.

To avoid cross-contamination problems between sample loadings, measurements were made by first analyzing the most dilute samples and then measuring successively larger sample quantities. Probe tips and micropipets were changed between samples and the $F A B$ probe tips were rinsed with dilute nitric acid followed by rinsing with high-purity water. Blank loadings $(1 \mu \mathrm{L}$ of methanol or water and $1 \mu \mathrm{L}$ glycerol) were measured as appropriate.

Surface tension measurements were made with a Fisher surface tensiometer (Fisher Scientific, Pittsburgh, PA), which operates according to the principles of operating devised by Du Nouy [16].

\section{Results and Discussion}

Not all sample preparations exhibit preferential analyte desorption (sample pulsing) at the initiation of bombardment. For many sample preparations the sample signal intensity rises to a steady and stable level. The aim of the SIM experiments was to determine the essential characteristics of preparation that cause samples to exhibit the pulsing effect. To gain an understanding of these characteristics a variety of analyte and matrix combinations were examined in the SIM mode. Figure 1 shows SIM data from a sample preparation that exhibits analyte pulsing. These data were obtained from a 5-nmol loading of erythromycin in glycerol. The lower trace shows the response from the protonated molecule of erythromycin at $m / z 734$, while the upper trace shows the response measured for the glycerol cluster ion at $m / z 737$. At time A the sample probe was rotated into the beam and sample bombardment commenced. This sample loading showed a pronounced pulse of analyte ions at the initiation of bombardment, while no such pulse was observed from the glycerol matrix. The pulse observed lasted for 200 to $300 \mathrm{~ms}$, after which the signal fell to a steady-state intensity. The sample was bombarded for $5 \mathrm{~s}$, then the probe was rotated out of the atom beam at time $B$. The sample was allowed to equilibrate for $10 \mathrm{~s}$ before being rotated back into the beam. Upon reintroduction the pulse was reproduced with similar intensity and duration to the first pulse. This effect was repeated for 12 cycles of sample introduction and removal with no 


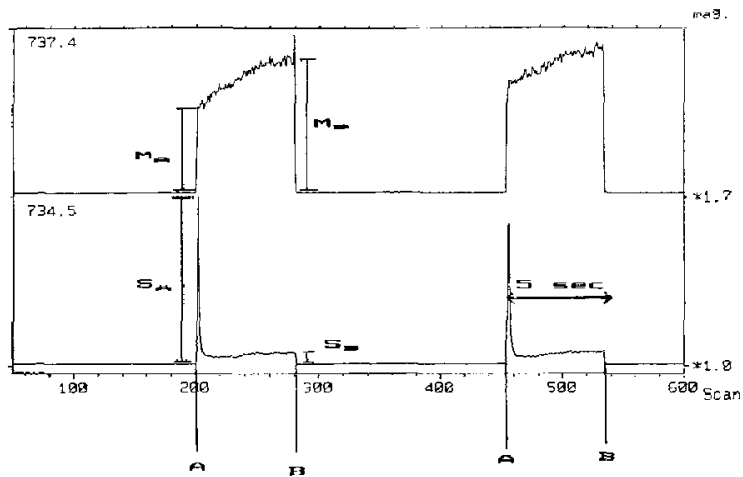

Figure 1. Selected ion monitoring traces for erythromycin pro tonated molecule (lower trace) and the proximate glycerol matrix ion. The sample is introduced into the atom beam at time $A$ and removed at time $B$. The relative heights of the sample and matrix ions at time A compared with their relative heights at time B indicate $G_{p r}$ the improvement in signal-to-chemical noise ratio resulting from sample pulsing.

significant change in the nature and magnitude of the signals.

The enhancement of the signal-to-chemical noise ratio due to pulsing may be estimated by measuring the relative intensities of analyte and matrix signals at bombardment initiation (position A) and comparing this with their relative intensities just prior to rotating the sample out of the beam (position $B$ ). Thus, the ratio of intensities at bombardment initiation, $R_{\mathrm{Ar}}$ is given by the relative intensities of the sample $\left(S_{A}\right)$ and matrix $\left(M_{\mathrm{A}}\right)$ signals. Similarly, the value $R_{\mathrm{B}}$ is given by the intensity ratio of the sample $\left(S_{\mathrm{B}}\right)$ and matrix $\left(M_{B}\right)$ at position $B$. The enhancement in signal-to-noise ratio due to pulsing may be expressed by a term $G_{p}$, which is given by the ratio $R_{\mathrm{A}} / R_{\mathrm{B}}$. A large numerical value of $G_{p}$ implies that pulsing yields a significant enhancement in signal-to-chemical noise ratio over the conventional FAB experiment, whereas a $G_{p}$ value of 1 implies no significant pulsing behavior.

We have studied the role of a number of factors and their influence on the value of $G_{p}$, concentrating upon the natures of the matrix and analyte, and the quantity of analyte loaded.

Figure 2 shows the effect of analyte quantity on the value of $G_{p}$ for erythromycin in glycerol. The maximum value of $G_{p}$ recorded was 18 , measured from a 500-fmol loading. At higher and lower sample loadings $G_{p}$ is diminished, but for different reasons. With larger sample loadings the analyte signal during normal bombardment increases, that is, the value $R_{\mathrm{B}}$ increases with sample loading. With smaller sample loadings the intensity of the sample ion pulses $\left(S_{A}\right)$ diminishes.

We have conducted similar experiments for other model compounds in glycerol, including other synthetic peptides, sugars, and classic surfactants. We observe that materials that would be expected to ex-

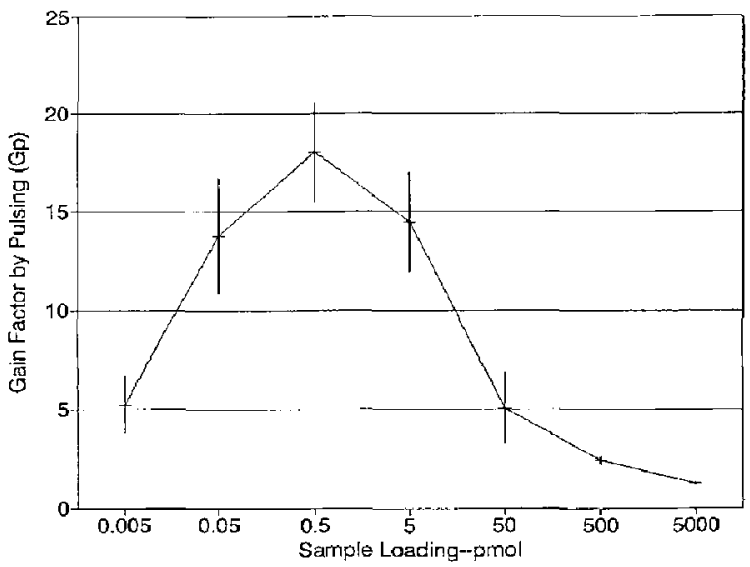

Figure 2. A plot of signal-to-matrix intensity ratio improvement $\left(G_{\mathrm{p}}\right)$ against sample loading for erythromycin in glycerol. Each plotted value is the mean of 12 measurements.

hibit surfactant properties in glycerol show pronounced pulsing behavior, and plots of the type shown in Figure 2 have similarly shaped curves. In contrast, sucrose is a very hydrophilic molecule that would not be expected to exhibit significant surfactant behavior. A plot of $G_{p}$ versus concentration for sucrose in glycerol is essentially a straight line with a $G_{p}$ value of 1 ; that is, sucrose does not exhibit pulsing behavior at any sample loading.

We have also examined pulsing behavior for the same range of analytes using NBA and thioglycerol matrices. In all cases except one these preparations exhibited behavior similar to that of sucrose in glycerol, that is, no pulsing behavior was observed at any concentration. The sole exception was a preparation of $C_{60}$ and $C_{70}$ fullerenes in NBA, which exhibited significant pulsing behavior.

This difference in pulsing behavior according to matrix selection we attribute to the differential surface tensions of the matrices. For pure glycerol the surface tension is very high, $63 \mathrm{dyn} \mathrm{cm}^{-1}$ at $17{ }^{\circ} \mathrm{C}$ [17]. Many glycerol-soluble compounds can reduce this surface tension by forming a surface layer, that is, by acting as surfactants. These preparations will be inclined to exhibit pulsed desorption activity. In the initial bombardment events this surface layer will be removed, resulting in a significant intensity of analyte ions, which we observe as the initial burst. Once the surface excess has been removed the analyte ion intensity will fall toward that observed in continuous bombardment. If the sample is removed from the atom beam the surface excess will regenerate as long as sufficient analyte and matrix are present.

In the absence of a literature value for the surface tension of NBA we measured a value for the Aldrichsupplied solvent of $35 \mathrm{dyn} \mathrm{cm}^{-1}$ at $21^{\circ} \mathrm{C}$ using a $\mathrm{Du}$ Nouy tensiometer [16]. This value is significantly lower than that for glycerol, which was measured as $60 \mathrm{dyn}$ 
$\mathrm{cm}^{-1}$ using the same equipment under the same conditions, and implies that many compounds that exhibit surface activity in glycerol will not do so in this matrix.

The term $G_{p}$ helps to explain the phenomenon we are attempting to exploit, and it is useful for investigating the physical chemistry of analyte-matrix systems. However, for a number of reasons this term does not truly reflect the sensitivity improvement that time-resolved array detection might afford. In particular, when measuring relative peak heights we compare measurements at some instant. In contrast, array detection allows for temporal integration of the ion current produced by the pulsed analyte signal. For erythromycin in glycerol the analyte protonated molecule could be barely observed by visual inspection in the conventional FAB mode at a sample loading of 10 pmol, while a pulsed signal for this ion was still clearly visible from low femtomole loadings. In terms of detection limits, these quantities are fairly typical for the peptides and other materials we have examined that exhibit good pulsed behavior in glycerol. These observations suggest a potential improvement in detection limit of three to four orders of magnitude for appropriate samples.

The limitations of conventional magnetic sector instruments with detection at a focal point have already been mentioned. The very short duration of the analyte pulse (200-300 ms) severely restricts their use. Furthermore, the absolute intensity of the analyte signal is changing constantly throughout the pulse. To take advantage of these pulses of ions in an analytical experiment requires a detector that will detect multiple masses in a concomitant and time-resolved fashion.

The data shown in Figure 3 were obtained using an array detector from a 400-amol loading of eledoisin in glycerol introduced into the atom beam in the same manner as for the SIM experiments. Immediately following exposure to the xenon atom beam the protonated molecule of eledoisin was observed at $\mathrm{m} / \mathrm{z}$ 1188.6 (Figure 3a). The subsequent spectra (Figure $3 b-e)$ were recorded at consecutive $100-\mathrm{ms}$ intervals following the first exposure. The signal for the proto nated molecule diminished in the second and third spectra and disappeared by the time the fourth spectrum was recorded. The glycerol cluster ion $[13 \mathrm{G}+$ $\mathrm{H}^{+}$appeared at $m / z \quad 1197.6$. The relative intensity of this signal increased until the fourth spectrum, at which point it is the only significant ion recorded. These data indicate that with a 400 -amol loading of eledoisin the analyte-to-matrix ion intensity ratio is significantly enhanced immediately following exposure to the fast atom beam and that the initial pulse of eledoisin parent ions persists for about $250 \mathrm{~ms}$. In contrast, a few picomoles of eledoisin were required to collect comparable data in the conventional FAB experiment. This represents a sensitivity enhancement of about four orders of magnitude, which is in keeping with expectations from the SIM experiments. We have observed

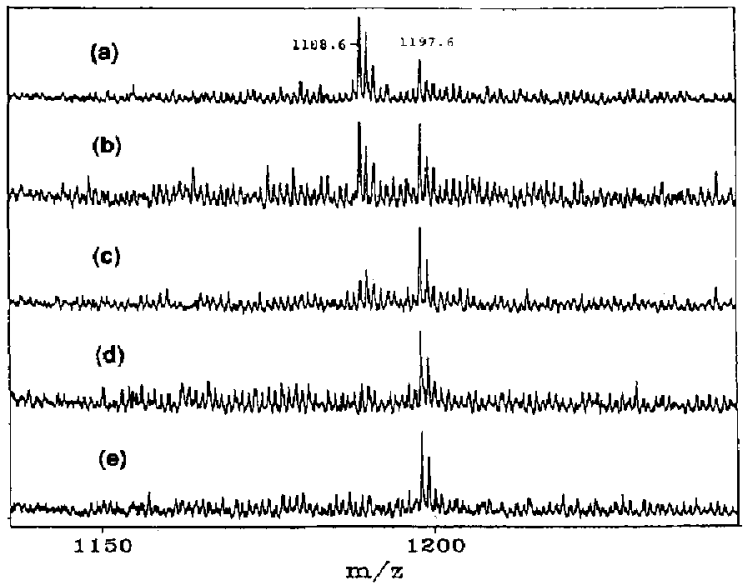

Figure 3. The differential ionization of eledoisin $(m / z$ 1188.6) and the glycerol cluster ion $[13 G+\mathrm{H}]^{+}(m / z$ 1197.6) recorded by array detection. The sample was a 400 amol loading of eledoisin in glycerol. Spectrum A was recorded when the probe was first rotated into the fast atom beam. Subsequent spectra (B-E) were recorded at successive $100-\mathrm{ms}$ intervals.

this behavior in a qualitative fashion for numerous other samples, for example substance $P$ and insulin, and the method appears to be widely applicable.

As expected from the SIM experiments, when the sample was rotated out of the atom beam and sufficient time allowed before reintroduction, then the pulse of sample ions may be repeated.

Figure 4 shows four spectra obtained from a single 400 -amol loading of eledoisin. The first spectrum was obtained with a fresh sample loading (Figure 4a). The second spectrum in Figure 4 was recorded after the probe had been rotated out of the beam for a $20-\mathrm{s}$

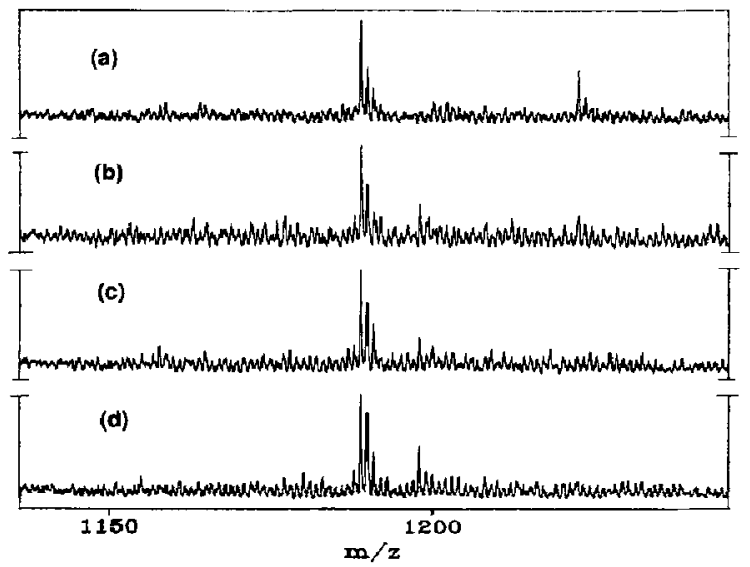

Figure 4. The pulsed effect from the same sample loading, showing its reproducible nature. The sample is 400 amol of eledoisin in glycerol. Spectrum A was obtained from the fresh loading. The remaining spectra were obtained from successive bombardment pulses with a 20-s interval between each pulse. 
interval following the recording of the first spectrum and then reintroduced. The remaining spectra were recorded following subsequent repetitions of this procedure. The ratios of analyte signal to chemical noise are quite similar in each spectrum.

A signal at $m / z 1221$ was observed in Figure 4a. The nature of this ion is uncertain. It is presumed to arise from Ultramark 1621, the mass calibrant used in this system. This ion was significant only in the first exposure of the sample to the atom beam.

We have observed that at smaller sample loadings a longer interval between bombardment pulses is required for repeated pulsing behavior to be observed. If the sample was rotated back into the beam too soon then the pulsing phenomenon was not observed. In this particular case, interpulse periods significantly shorter than $20 \mathrm{~s}$ resulted in spectra showing only the glycerol cluster ion at $m / z$ 1197.6.

These observations support the hypothesis that sample diffusion from the bulk to the surface of the matrix is important in determining the quality of FAB/LSIMS spectra. The role of diffusion processes has been discussed at some length in the literature [4, 18-20].

The phenomenon of sample suppression, whereby the high surface activity of one or more components of a mixture subjected to $\mathrm{FAB}$ excludes other components from the surface, has been widely studied and discussed [5]. Pulsed ionization coupled with array detection provides some interesting insights into this phenomenon. Figure 5 shows a pulsed FAB experiment conducted on a sample of synthetic peptide prepared in glycerol. This sample also contained a proportion of the peptide retaining a $t$-butyl protecting group on one of the hydroxylic peptide side chains. This component

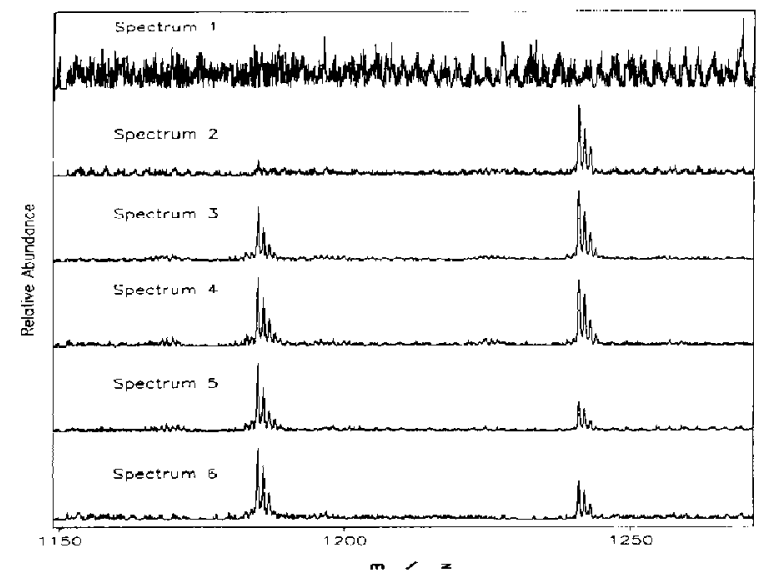

Figure 5. Six successive molecular region spectra from a mixture of the peptide KLGEA YNQMM (MW 1183) and a derivative containing a t-butyl function (MW 1139), recorded by pulsed bombardment and array detection. Spectrum 1 was recorded immediately prior to sample introduction. Spectra were integrated for $65 \mathrm{~ms}$ with a $35-\mathrm{ms}$ array refresh between spectra. Each spectrum is normalized to itself. was present as the result of incomplete deprotection after synthesis. The six spectra in Figure 5 represent a time course study of the events that occur in the first $500 \mathrm{~ms}$ following the commencement of bombardment. The spectra are recorded at $100-\mathrm{ms}$ intervals. Spectrum 1 was recorded immediately prior to sample introduction. Immediately following exposure to the atom beam (spectrum 2) the signal for the component retaining the $t$-butyl function was predominant. In the subsequent spectra the relative intensity of the free peptide signal increased, until by spectrum 6 the intensity of the protected peptide was about $50 \%$ relative to the unprotected peptide. This relative intensity was very similar to that observed in the normal FAB experiment.

Our interpretation of these data is as follows. The $t$-butyl functional group confers additional hydrophobicity to the molecule; hence this component would be expected to have superior surface activity compared to the free peptide. Therefore in the fresh sample preparation this component dominates the sample surface. This assertion is evidenced by the fact that in the spectrum recorded immediately following bombardment initiation only the derivatized component was observed. Following the initial bombardment of the surface layer the two forms of this peptide migrate towards the surface. The $t$-butyl-containing component has superior surface activity and therefore would be expected to reestablish a surface layer. However, this new surface material is continuously removed as long as bombardment continues. In this instance the more hydrophobic component is present in insufficient quantity to form a surface layer at the equilibrium bombardment condition. The less hydrophobic component, in this case the free peptide, is then observed in the later spectra (Figure 5, spectra 3-6).

It is interesting to note that the relative abundance of these two components established by reversed-phase high-performance liquid chromatography coupled with UV absorption is quite different to that implied by FAB mass spectrometry. By the former method the relative abundance of the protected peptide was about $6 \%$ of that of the unprotected peptide, contrasting with about $50 \%$ relative intensity observed in the mass spectra. This marked effect of the additional $t$-butyl group upon the FAB spectra provides an illuminating example of the relatively small structural changes required for the exhibition of the sample suppression phenomenon [5].

\section{Conclusion}

In designing a pulsed bombardment experiment many factors need to be considered. In particular, there are many variables affecting the formation of a pulsed beam. The rise time of the atom gun power supply, the rare-gas pressure in the atom gun, and the duration of the pulse are some of the important considerations. In this study we have attempted to avoid the effects of 
these variables by modeling a system in which the source of bombarding particles is operating in a steady state. The mechanical movement of the sample serves to accurately define the time at which sample bombardment commences, independent of the problems associated with the formation of a properly regulated pulsed bombarding atom beam.

The results presented here show that coupling pulsed sample bombardment with the time-dependent array detector appears to provide a method of enhancing FAB sensitivity for certain analytes. This enhancement relies on an ability to exploit solution chemistry. Materials that would be expected to act as surfactants, that is, to decrease surface excess energy, in FAB matrices exhibit pulsing behavior. We have studied a wide variety of peptides and classic surfactants. All of these exhibit pulsing behavior when prepared in glycerol, but none did so when prepared in NBA. Array detection afforded an improvement in detection limit for the glycerol preparations that was typically three to four orders of magnitude better than that observed with conventional FAB for the same preparation. $\mathrm{Hy}$ drophilic materials, such as small sugars, did not exhibit pulsed behavior in any matrix and array detection did not afford any sensitivity improvement for these samples.

The effects that varying the atom beam pulse characteristics have on the analyte ion pulse have not been determined. It is anticipated that the atom beam energy, flux, and pulse duration will each play a significant role in determining the desorption characteristics of the analyte ions.

Finally, it is important to realize that the array detector integrates signals for some preset time period. Optimal performance of the pulsed FAB system will require synchronization of the data integration, array reset, and array refresh periods with the bombardment pulse. The circuitry required to perform this synchronization is under development.

\section{Acknowledgment}

We acknowledge Brian $\Gamma$. Mehlman for his technical assistance.

\section{References}

1. Barber, M.; Bordoli, R. S.; Sedgwick, R. D.; Tyler, A. N. I. Chem. Soc., Chem. Commun. 1981, 325.

2. Aberth, W.; Straub, K. M.; Burlingame, A. L. Anal. Chem. 1982, 54, 2029.

3. Barber, M.; Bordoli, R. S.; Elliott, G. J.; Sedgwick, R. D.; Tyler, A. N. Anal. Chem. 1982, 54,645A.

4. Barber, M.; Bordoli, R. S.; Elliott, G. J.; Sedgwick, R. D.; Tyler, A. N. J. Chem. Soc., Faraday Trans. 1 1983, 79, 1249.

5. Ligon, W. V. Jr. In Biological Mass Spectrometry; Burlingame, A. L.; McCloskey. J. A., Eds.; Elsevier: Amsterdam, 1990; P 61.

6. Ligon, W. V. Jr.; Dorn, S. B. J. Am. Chem. Soc. 1988, 110, 6684.

7. Ligon, W. V. Jr. Int. J. Mass Spectrom. Ion Proc. 1984, 61, 113.

8. Tecklenburg, R. E. Jr.; Castro, M. E.; Russell, D. H. Anal. Chem. 1989, 61, 153

9. Hunt, D. F.; Shabanowitz, J.; Yates, J. R. III; Zhu, N. Z; Russell, D. H.; Castro, M. E. Proc. Natl. Acad. Sci. U.S.A. 1987, 84,620 .

10. Olthoff, J. K.; Honovich, J. P,; Cotter, R. J. Anal. Chem. 1987, $59,999$.

11. Olthoff, J. K; Cotter, R. J. Nucl. Instrum. Meth. Phys. Res. $1987, B 26,566$.

12. Musselman, B. D.; Stults, J. T.; Holland, J. F. Proceedings of the 35 th ASMS Conference on Mass Spectrometry and Allied Topics, Denver, CO, May 24-29, 1987; p 302.

13. Ouwerkerk, C. E. D.; Boerboom, A. J. H.; Matsuo. T; Sakurai, T. Int. J. Mass Spectrom. Ion Proc. 1986, 70, 79.

14. Ashcroft, A. E.; Brown, R. S.; Coles, A. D.; Evans, S.; Milton, D. J.; Wright, B. Spectroscopy 1987, 3, 57.

15. Hill, J. A.; Biller, J. E.; Martin, S. A.; Biemann, K.; Yoshidome, K, Sato, K. Int. J. Mass Spectrom. Ion Proc. 1989, 92, 211.

16. Du Nouy, P. J. Gen. Physiol. 1919, 1, 521.

17. Lange's Handbook of Chemistry, 13th ed.; Dean, J. A., Ed.; McGraw-Hill: New York, 1985.

18. Ligon, W. V. Jr.; Dorn, S. B. Int. J. Mass Spectrom. Ion Proc. $1994,57,75$.

19. Todd, P. J. J. Am. Soc. Mass Spectrom. 1991, 2, 33.

20. Todd, P. J. Org. Mass Spectrom. 1988, 23, 419. 